\title{
The System for Control and Stabilization of the Beam Position in the Microtron MT-25 in Prague
}

\author{
Č. Šimáně, M. Vognar, D. Chvátil
}

\begin{abstract}
A method of control the beam position at crucial points of the transport system and for the stabilization of its output position has been proposed and preliminary tested. The method is based on secondary electron emission from a thin metallic wire probe induced by electrons from the $25 \mathrm{MeV}$ microtron. It was demonstrated, that magnetic field of the order of $0.2 \mathrm{~T}$ and parallel to the wire probe in front of the orifice of the extraction channel in the acceleration space, does not prevent the functioning of the method. A strong parasitic effect of secondary electron emission from the material of the channel and its support construction was found, leading to the inversion of the electron current polarity from the wire. This effect can be to great extent eliminated by negative electric potential bias relative to the channel. At the electron output current of $1 \mu \mathrm{A}$ the secondary emission current from the wire probe of $0.3 \mathrm{~mm}$ diameter is of the order of several nA. Two electromechanical systems were designed for the removal of the probes from the beam path, to avoid the deterioration of the electron beam quality by scattering. Electronic schemes used for remote measurement of small probe currents, suppressing the influence of strong electromagnetic noise, are described. For stabilization of the output beam position two wire probes situated in air close to the Al output window were used. These probes having been placed at the periphery of the beam did not deteriorate the beam quality. The difference of their emission currents was used as an error signal to control the magnetic field of the last dipole, which kept the beam in the center of the output window.
\end{abstract}

Keywords: microtron, electron transport, beam detection, beam position stabilization, secondary electron emission.

\section{Introduction}

The electron beam transmission system in the Prague MT-25 microtron requires accurate alignment of the beam with all deflecting or focusing magnetic elements. Without knowledge of the beam position at crucial points of the transport system, the alignment is a troublesome operation even for an experienced operator. Therefore it is desirable to have actual knowledge about the beam position, which however must be obtained without any deterioration of the electron beam quality. Once the alignment is obtained, the main duty of the operator is to hold the beam in the center of the output window. This function, requiring permanent attention from the side of the operator, can be automated as well.

There exist several types of beam non-disturbing position detection systems. In our case we decided to apply the system based on secondary electron emission from impact of accelerated electrons on thin metallic wire probe. The amount of energy dissipated in a wire probe is negligible and no supplementary cooling of the wire is necessary. The deterioration of the beam by scattering on a single wire is very small; nevertheless it must be taken into account, if several wires in series are placed in the beam path. Therefore the wire probes can be used only in the period of the alignment of the beam and should be removed from the beam path after the alignment has been accomplished. Only probes situated at the beam periphery can stay at place permanently.

\section{Experimental part}

\subsection{Stabilization of the beam position at the output window}

The wire probe method was tested in connection with the stabilization of the beam position at the output. To be sure, that the method will work in vacuum as well as in air, an electrically isolated copper wire $\phi 0.4 \mathrm{~mm}$ was placed in the axis of a thin wall cylindrical aluminum chamber of $28 \mathrm{~mm} \mathrm{ID,} \mathrm{which}$ could be evacuated. The chamber wall was grounded and a load resistor was inserted between the wire and the ground. The potential on the resistor was measured by a low noise, low drift instrumental amplifier. The chamber was exposed to the electron beam with the wire in the beam axis and evacuated. The portion of the $0.8 \mu \mathrm{A}, 22 \mathrm{MeV}$ electron beam passing through the wire was estimated to $0.15 \mu \mathrm{A}$. The total beam intensity was monitored by an induction pick up system [1] at the end of the electron transmission line. The wire potentials, measured on load resistors going from $10^{5}$ to $10^{6} \mathrm{ohms}$, were in the $\mathrm{mV}$ range. Currents calculated from potentials and resistor values were in all cases $0.08 \mu \mathrm{A}$, independent on the resistor values. The loss of intensity of the primary beam at passage through the wire being negligible, the current is due exclusively to secondary electrons leaving the wire and reaching the wall of the chamber. The wire is charged positively and behaves as a constant current source.

The situation changed, when the same measurements were made at atmospheric air pressure in the chamber. In this case several effects must be taken into account. Most of the secondary electrons, with an energy spectrum from fraction of $\mathrm{eV}$ and more, cannot reach the wall of the chamber. Their energy is dissipated in collisions with the nitrogen and oxygen atoms or molecules and finally, captured by them, they form negative atomic or molecular ions. A negative space charge barrier built up in the space between the wire and the wall of the chamber prevents low energy secondary electrons to escape from the region in vicinity of the wire. The diffusion of the space charge both toward the wire and the chamber represents additional currents. At atmospheric pressure, the backward current of negative ions toward the central wire overweighs the current toward the wall of the chamber. Con- 


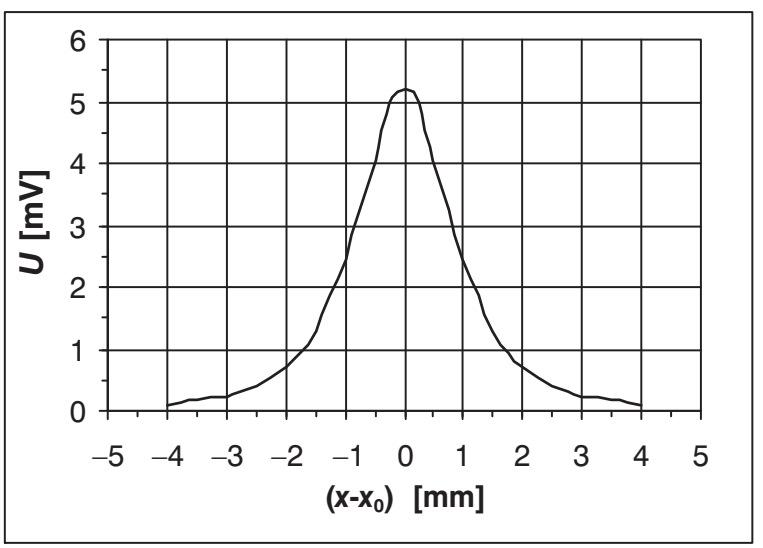

Fig. 1: Potential on the load resistor $R=10^{5} \Omega$ of the wire probe versus the horizontal beam departure from the axis

tact potentials between the $\mathrm{Al}$ chamber and $\mathrm{Cu}$ wire as well as thermoelectric potentials may also in some way modify the load resistor current. In detailed analysis, the pulse character of the microtron electron beam should be also taken into account. Each $2.5 \mu$ seam pulse is followed by a $2.5 \mathrm{~ms}$ pause, during which the space charge diffuses without being nourished by secondary electrons. Some role may also play the ionization of air primary beam. The observed final result of all these rather complicated effects is the reduction of the current in the load resistor as compared with the current in the evacuated chamber. When keeping the load resistor below $100 \mathrm{k} \Omega$, the observed reduction represents about $50 \%$.

Based on these experimental results, a system for stabilizing the beam in the horizontal plane was developed [2]. In its simplest version, two parallel $0.4 \mathrm{~mm} \mathrm{Cu}$ wire probes (E1 and E2) were placed in air vertically in front of the exit window of the electron transport line, symmetrically to its center. The $6 \mathrm{~mm}$ distance between them was selected as suitable for normally adjusted $3 \mathrm{~mm}$ beam half-width. For this probe configuration, using measured values from Fig. 1, the difference $U 2-U 1$ of potentials on the load resistors (error signal) as function of the beam departure from the central position was calculated (Fig. 2).

The probes were connected to the inputs of a differential instrument amplifier (Fig. 3) through a frequency filter suppressing the pulse character of the secondary electron emission currents and environmental electromagnetic noise. The inherent noise of the amplifier was reduced to units of $\mu \mathrm{V}$, which corresponds to a noise current of the order of $10 \mathrm{pA}$ at

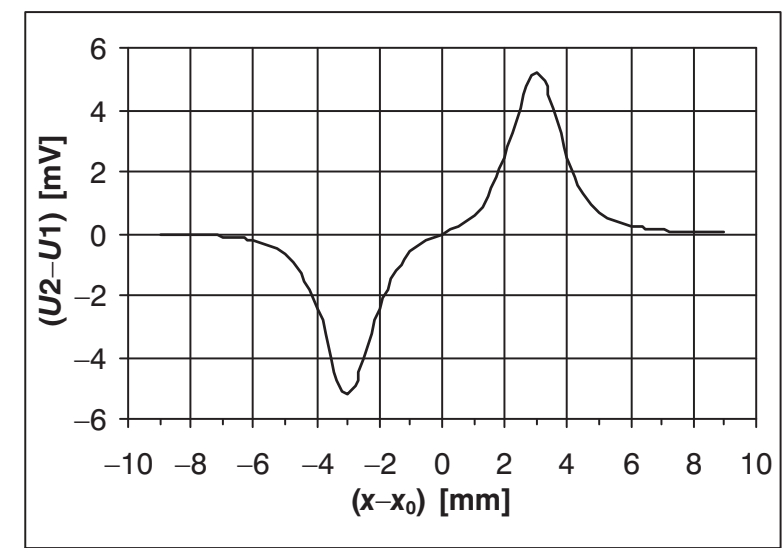

Fig. 2: Calculated values of the error potential difference between the load resistors $R=10^{5} \Omega$ of two wire probes versus the horizontal beam departure from the axis (at $3 \mathrm{~mm}$ beam half width)

the input. The output of the instrument amplifier was connected to a power amplifier feeding the auxiliary dipole winding for fine beam position control. In such a way the error signal controls the dipole magnetic field, which compensate the departure of the beam from the central position. In our case, 0.28 A current in the auxiliary winding is necessary to compensate $1 \mathrm{~mm}$ departure. The current in the auxiliary dipole winding as function of the beam departure from the symmetry plane of the wire probes at $1 \mu \mathrm{A}$ mean beam current is presented on Fig. 4. The stabilization factor at $1 \mu \mathrm{A}$ mean beam current, defined as the ratio of the beam departure from the center without and with stabilization, was about 20 , increasing linearly with the beam current.

\subsection{Tracing of the beam path in the transport system}

Preliminary experiments were carried out with the aim to verify the applicability of this system for alignment of the iron channel in the acceleration space, through which the electrons are extracted. The electron energy during these experiments was fixed to $22 \mathrm{MeV}$. In correct position, the axis of the channel must be oriented tangentially to the electron orbit and the center of its input orifice must coincide with the point of contact with the orbit. To find this position, an electrically isolated stainless iron wire probe $\phi 0.3 \mathrm{~mm}$ was fixed vertically on the channel, crossing its axis in a distance of $15 \mathrm{~mm}$ in front of the input orifice. The secondary electron emission

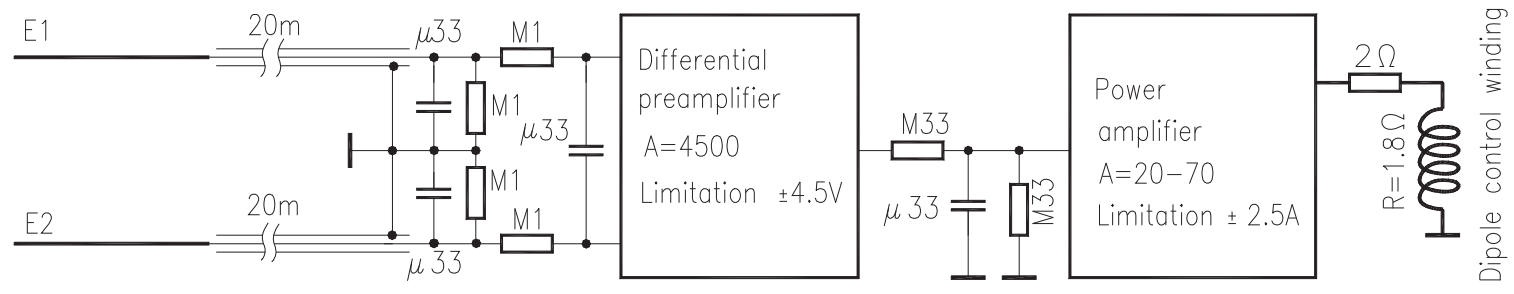

Fig. 3: Principal electronic scheme of the stabilization device 


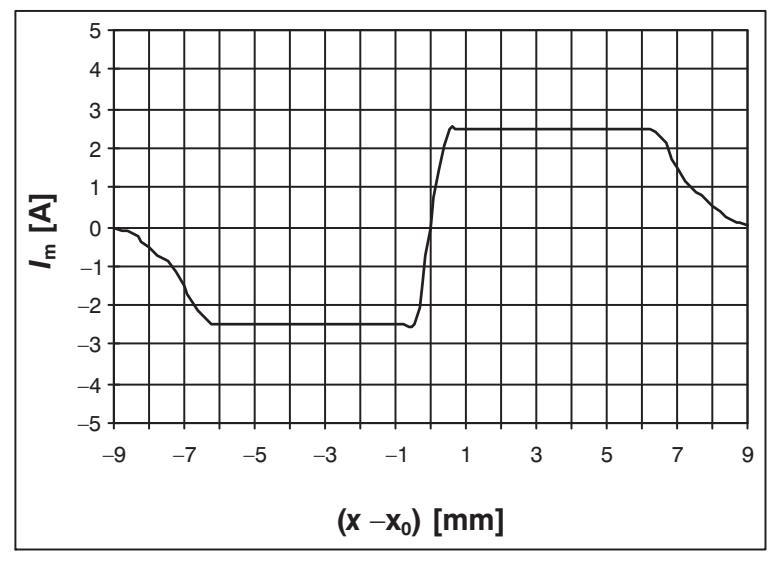

Fig. 4: Compensation current in the auxiliary dipole winding versus the horizontal beam departure from the axis

current of the probe was measured as function of the channel position relative to the electron orbit.

The experiment furnished two important results. First, it was confirmed, that magnetic field existing in the acceleration space of the order of $0.2 \mathrm{~T}$ and parallel to the wire probe, does not prevent its functioning, which was not at all evident in advance. Secondly, a strong parasitic effect, leading to the inversion of electron current direction from the wire probe, was observed, the origin of which was the secondary electron emission from the channel and its support material hit by the beam. The current of the probe is thus an algebraic sum of its own secondary emission current and of current of secondary electrons emitted from the support material and collected by the probe. As seen from Fig. 5, the setting of the wire probe on negative bremspotentials $-9,-18$ and $-27 \mathrm{~V}$ against the channel potential inhibited progressively the collection of secondary electrons from the construction material. At a bremspotential of $-27 \mathrm{~V}$ the maximum of the current peak was clearly identifiable and current inversion to great extent suppressed.

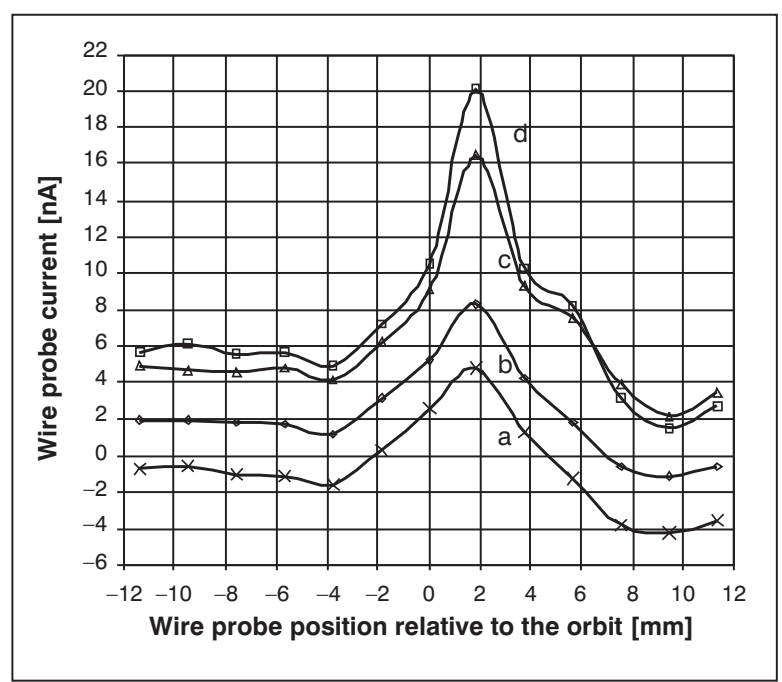

Fig. 5: Current of the wire probe in front of the input orifice of the extraction channel as function of its departure from the electron orbit
In next experiments, the possibility of using the system was verified for finding the correct channel position at passage from an electron orbit to the next (to the previous) one. During the passage, the effect of secondary emission from the channel and its support material was very strong and could not be fully suppressed even if the bremspotential was used. From the same reason the determination of the correct channel position by differential wire probes, which has been tried too, gave not satisfactory results. Nevertheless, as can be seen from Fig. 6, using only a single wire probe the correct channel position on two neighbor orbits can be found unambiguously.

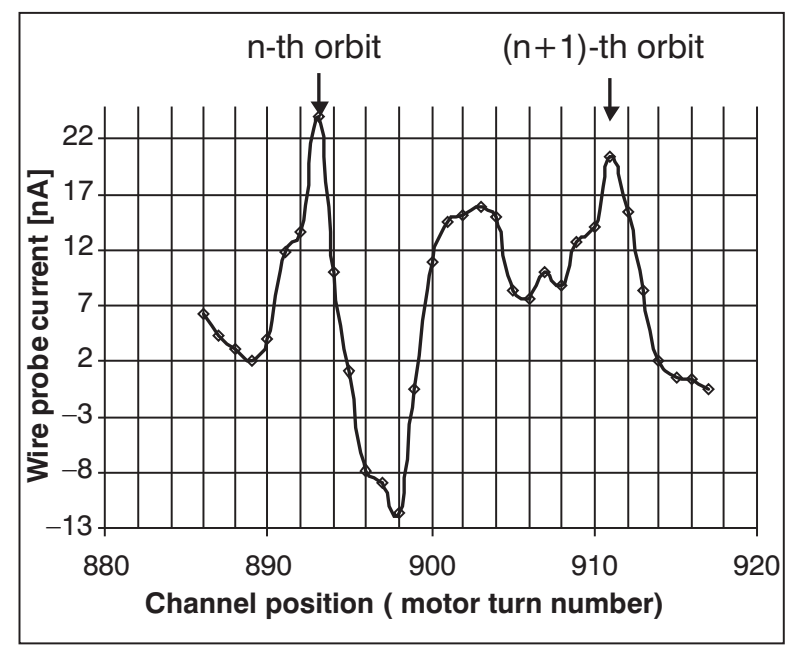

Fig. 6: The current of the wire probe in front of the input orifice of the extraction channel in course of the passage from the $n^{\text {th }}$ to the $(n+1)^{\text {th }}$ electron orbit (bremspotential of the wire probe equals to $-27 \mathrm{~V}$ )

The experiments gave also a crude estimate of the order of magnitude of the secondary emission current. The $\phi 0.3 \mathrm{~mm}$ wire probe was situated in front of the orifice of the iron channel, where the horizontal width of the beam is about $6 \mathrm{~mm}$. Setting the accelerated electron output current to $1 \mu \mathrm{A}$, the maximum of the secondary electron current was of the order of $20 \mathrm{nA}$. Because of the unknown distribution of the current density in the beam the coefficient of electron secondary emission could not be calculated and compared with the experimental results, so that this value is only informative. A low noise low drift analog amplifier has been built working in two wire current loop with the measuring instrument on the panel in the remote control room. The amplifier was placed in vicinity of the accelerator at a place with relatively low radiation level to avoid its deterioration by radiation. In this way the influence of strong electromagnetic noise in the microtron room on measurement of the small probe currents was effectively suppressed.

The supposed deterioration of the beam quality by scattering on the wire probe was experimentally confirmed. Therefore a system has been designed, which will be used in the future electron transmission line, with wire probes, which can be removed from the beam after the alignment procedure has been accomplished. It is supposed to place the wire probes at the inputs of the magnetic quadrupole lenses and deflection dipoles, both to align the horizontal and vertical positions of the beam. Two wire probes for setting the horizontal and vertical central positions of the beam will 


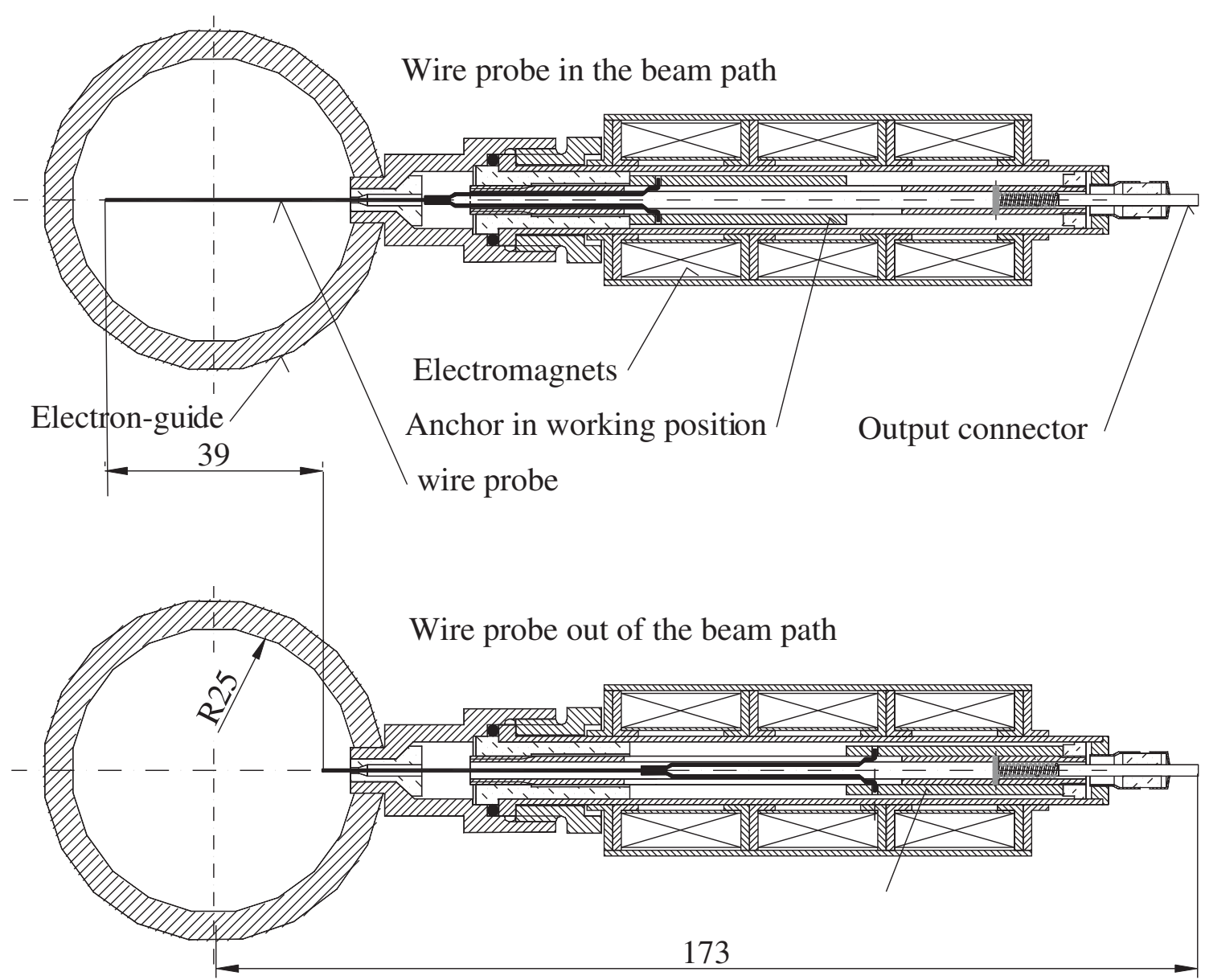

Fig. 7: Linear shift mechanism for setting the wire probe in and out of the electron beam path

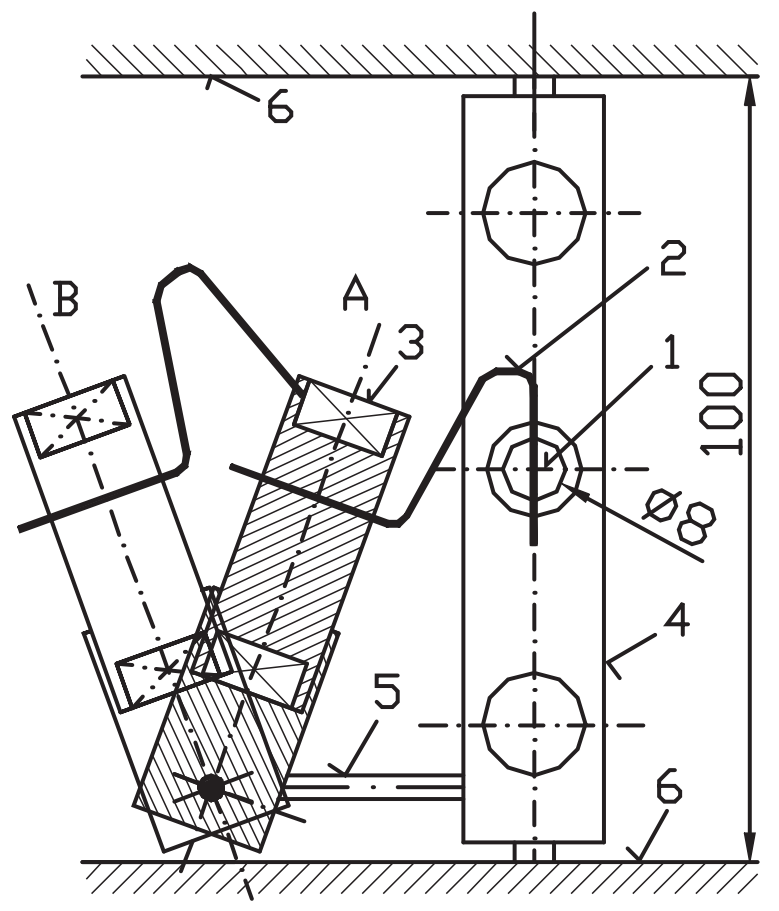

Fig. 8: Flipping coil mechanism for setting the wire probe in front of the electron extraction channel and to withdraw it: 1-extraction channel input orifice, 2-wire probe, 3-coil, 4-holder of the extraction channel, 5 -shaft of the flipping coil holder, 6-pole pieces of the microtron electromagnet be oriented perpendicularly to the transmission line axis and will be made extractable from the beam. For this purpose a special mechanism was designed securing the linear transport of the probe by external magnetic fields (Fig. 7). Additional differential wire probes will be situated in the beam transmission line at the periphery of the beam, serving for determination of the sign of deviation of the beam from the axis. Being situated at the periphery of the beam, they will perturb but insignificantly the beam and can be fixed permanently in the transmission line. The wire probe in front of the orifice of the extraction channel is fixed on a coil with two stable positions. In one stable position the wire is in front of the channel orifice, in the other position the probe is removed from the beam path (Fig. 8). Current pulses of appropriate polarity actuate the flipping of the coil from one to the other position when magnetic field in the acceleration space is on. The system was preliminary tested inside the acceleration space of the microtron with satisfactory results.

\section{Conclusion}

The use of the secondary electron emission from wire probes represents an advantageous way of electron beam position control when setting the parameters of magnetic deflecting and focusing elements of the electron transport system. The differential wire probes can be used for automatic stabilization of the output beam position. Introduction of 
these probes in the transport system in the way described does not deteriorate the beam quality. No additional cooling system is required, what represents a very important feature of the wire probes. The incorporating of the beam position control in the existing transport system is expected to result in optimization of its parameters, improvement of the output beam quality and simplification of the overall microtron control.

\section{References}

[1] Šimáně Č., Vognar M.: Induction Pick Up System For Microtron MT25 Current Measuring and Position Indication. Šimáně, Č., Vognar, M.: Czech Technical University in Prague, Workshop 98, Prague, 1998.
[2] Šimáně Č., Vognar M., Němec V.: Stabilization of microtron electron beam position. Czech Technical University in Prague, Workshop 2000, Part B, Prague, 2000, p. 540.

Prof. Ing. Čestmír Šimáně, DrSc.

Ing. Miroslav Vognar

Ing. David Chvátil

Department of Dosimetry and Application of Ionizing Radiation

Czech Technical University in Prague

Faculty of Nuclear Sciences and Physical Engineering Břehová 7

11519 Praha 1, Czech Republic 